\title{
A Structure-Sharing Representation for Unification-Based Grammar Formalisms
}

\author{
Fernando C. N. Pereira \\ Artificial Intelligence Center, SRI International \\ and \\ Center for the Study of Language and Information \\ Stanford University
}

\begin{abstract}
This paper describes a structure-sharing method for the representation of complex phrase types in a parser for PATR-II, a unification-based grammar formalism.

In parsers for unification-based grammar formalisms, complex phrase types are derived by incremental refinement of the phrase types defined in grammar rules and lexical entries. In a naive implementation, a new phrase type is built by copying older ones and then combining the ropies acrording to the constraints stated in a grammar rule. The structure-sharing method was designed to eliminate most silch copying; indeed, practical tests suggest that the use of this technique reduces parsing time by as much as $60^{\circ} \%$.

The present work is inspired by the structure-sharing method for theorem proving introduced by Boyer and Moore and on the variant of it that is used in some Prolog implemintations.
\end{abstract}

\section{Overview}

In this paper I describe a method, structure sharing, for the representation of complex phrase types in a parser for PATR-II, a unification-based grammar formalism.

In parsers for unification-based grammar formalisms, complex phrase types are derived by incremental refinement of the phrase types defined in grammar rules and lexical entries. In a naive implementation, a new phrase type is built by copying older ones and then combining the copies acording to the constraints stated in a grammar rule. The structure-sharing method eliminates most such copying by

This research, made possible in part by a gitt from the Systums Development Foundation, was also supported by the Detense Advaoced Research Projects Agency under Contracts N00039-80-C-05.75 and Non039-84-C-0.524 with the Naval Electronic Systems Command. The views and conclusions contained in this document are those of the author and should not be interpreted as representative of the official policies, either expressed or implied. of the Defense Advanred Researrh Projects Agency, or the United States government.

Thanks are due to Stuart Shieber, Lauri harttunen, and Ray Perrault for their comments on earlier preyentations of this ntaterial. representing updates to objects (phrase types) separately from the objects themselves.

The present work is inspired by the structure-sharing method for theorem proving introduced by Boyer and Moore [1] and on the variant of it that is used in some Prolog implementations [9].

\section{Grammars with Unification}

The data representation discussed in this paper is applicable, with but minor changes, to a variety of grammar formalisms based on unification, such as definite-clause grammars [6], functional-unification grammar $|4|$, lexical-functional grammar $[2]$ and PATR-II $[8]$. For the sake of concreteness, however, our discussion will be in terms of the PATR-II formalism.

The basic idea of unification-baserl grammar formalisms is very simple. As with context-free grammars. grammar rules state how phrase types combine to yield other phrase types. But whereass a context-free grammar allows only a finite number of predefined atomic phrase types or nonterminals. a unification-based grammar will in general define implicitly an infinity of phrase types.

A phrase type is defined by a set of constraints. A grammar sule is a set of constraints betweon the type lis of a phrase and the types $\Gamma_{1} \ldots . X_{n}$ of its eomstituents. The rule may lee applieel to the allalysis of at wring se as the concatenation of constiturents $s_{1} \ldots s_{n}$ if and only if the types of the $s_{i}$ are compatible with the lypes $I_{i}$ and the constraints in the rule.

linification is the operation that determines whether two types are compatuble by building the most. general type compatible with both.

If the constraints are equations between attributes of phrase types, as is the ase in PXTR-II. Iwo phrase lypen

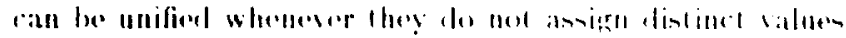

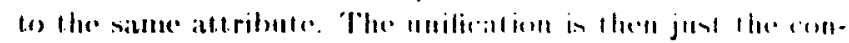
junction (set union) of the corresponding sets of constraints [5].

Ilere is a sample rule, in a simplified version of the PATR- 
II notation:

$$
\begin{aligned}
& X_{0} \rightarrow X_{1} X_{2}:\left\langle X_{0} \text { cat }\right\rangle=S \\
& \left\langle X_{1} \text { cat }\right\rangle=N P \\
& \left\langle X_{2} \text { cat }\right\rangle=V P \\
& \left\langle X_{1} \text { agr }\right\rangle=\left(X_{2} \text { agr }\right\rangle \\
& \left\langle X_{0} \text { trans }\right\rangle=\left(X_{2} \text { trans }\right) \\
& \text { ( } \left.X_{0} \text { trans arg }\right)=\left(X_{1} \text { trans }\right)
\end{aligned}
$$

This rule may be read as stating that a phrase of type $X_{0}$ can be the concatenation of a phrase of type $X_{1}$ and a phrase of type $X_{2}$, provided that the attribute equations of the rule are satisfied if the phrases are substituted for their types. The equations state that phrases of types $X_{0}, X_{1}$, and $X_{2}$ have categories $S, N P$, and $V P$, respectively, that types $X_{1}$ and $X_{2}$ have the same agreement value, that types $X_{0}$ and $X_{2}$ have the same translation, and that the first argument of $X_{0}$ 's translation is the transiation of $X_{1}$.

Formally, the expressions of the form $\left(l_{1} \ldots l_{m}\right)$ used in attribute equations are paths and each $l_{i}$ is a label.

When all the phrase types in a rule are given constant cat (category) values by the rule, we can use an abbreviated notation in which the phrase type variables $X_{i}$ are replaced by their category values and the category-setting equations are omitted. For example, rule (1) may be written as

$$
\begin{aligned}
& S \rightarrow N P V P:\langle N P \text { agr }\rangle=\langle V P \text { agr }\rangle \\
& \text { (S trana) }=\text { (VP trans) } \\
& \langle S \text { trans arg }\rangle=\text { (NP trans) }
\end{aligned}
$$

in existing PATR-II implementations, phrase types are not actually represented by their sets of defining equations. Instead, they are represented by symbolic solutions of the equations in the form of directed acyclic graphs (dags) with arcs labeled by the attributes used in the equations. Dag nodes represent the values of attributes and an arc labeled by $l$ goes from node $m$ to node $n$ if and only if, according to the equations, the value represented by $m$ has $n$ as the value of its $/$ attribute [5].

A dag node (and by extension a dag) is said to be atomic if it represents a constant value; complex if it has some outgoing arrs; and a leaf if is is neither atomic or complex, that is, if it represents an as yet completely undetermined value. The domain dom(d) of a complex dag $d$ is the set of labels on arcs leaving the top node of $d$. Given a dag $d$ and a label $l \in \operatorname{dom}(d)$ we denote by $d / l$ the subdag of $d$ at the end of the arc labeled $l$ from the top node of $d$. By extension, for any path $p$ whose labels are in the domains of the appropriate subdags, $d / p$ represents the subdag of $d$ at the end of path $p$ from the root of $d$.

For uniformity, lexical entries and grammar rules are also represented by appropriate dags. For example, the dag for rule (1) is shown in Figure 1.

\section{The Problem}

In a chart parser [3] all the intermediate stages of derivations are encoded in edges, representing either incomplete

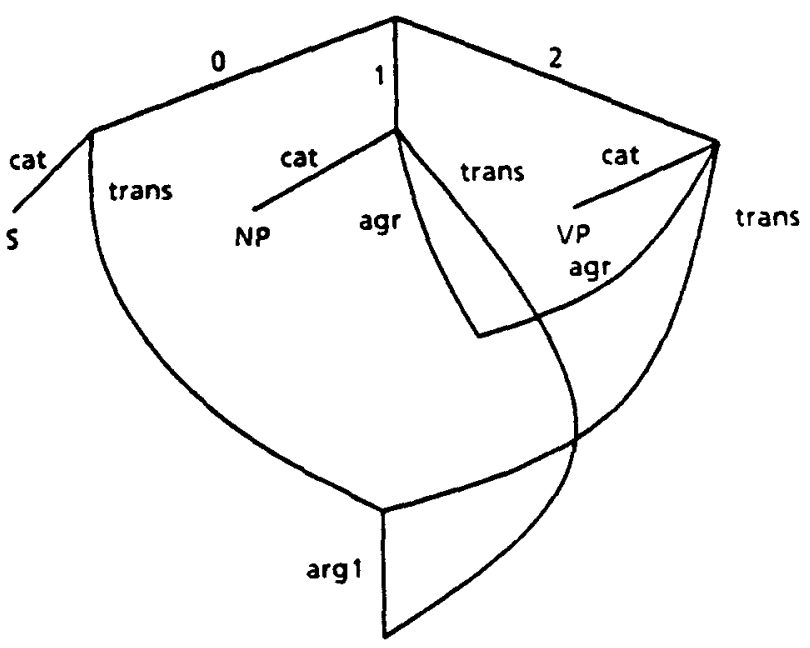

Figure 1: Dag Representation of a Rule

(active) or complete (passive) phrases. For PATR-II, each edge contains a dag instance that represents the phrase type of that edge. The problem we address here is how to encode multiple dag instances efficiently.

In a chart parser for context-free grammars, the solution is trivial: instances can be represented by the unique internal names (that is, addresses) of their objects because the information contained in an instance is exactly the same as that in the original object.

In a parser for PATR-Il or any other unification-based formalism, however, distinct instances of an object will in general specify different values for attributes left unspecified in the original object. Clearly, the attribute values specified for one instance are independent of those for another instance of the same object.

One obvious solution is to build new instances by copy. ing the original object and then updating the copy with the new attribute values. This was the solution adopted in the first PATR-II parser [8]. The high cost of this solution both in time spent copying and in space required for the copies themselves constitutes the principal justification for employ. ing the method described bere.

\section{Structure Sharing}

Structure sharing is based on the observation that an initial object, together with a list of update records, contains the same information as the object that results from applying the updates to the initial object. In this way, we can trade the cost of actually applying the updates (with possible copying to avoid the destruction of the source object) against the cost of having to compute the effects of updates when examining the derived object. This reasoning applies in particular to dag instances that are the result of adding attribute values to other instances. 
As in the variant of Boyer and Moore's method [1] used in Prolog [9], I shall represent a dag instance by a molecule (see Figure 2) consisting of

1. [A pointer to] the initial dag, the instance's skeleton

2. [A pointer to] a table of updates of the skeleton, the instance's environment.

Environments may contain two kinds of updates: reroutings that replace a dag node with another dag; are bindings that add to a node a new outgoing arc pointing to a dag. Figure 3 shows the unification of the dags

$$
\begin{aligned}
& I_{1}=[a: x, b: y] \\
& I_{2}=[c:[d: e]]
\end{aligned}
$$

After unification, the top node of $I_{2}$ is rerouted to $I_{1}$ and the top node of $I_{1}$ gets an arc binding with label $c$ and a value that is the subdag [ $d: e]$ of $I_{2}$. As we shall see later, any update of a dag represented by a molecule is either an update of the molecule's skeleton or an update of a dag (to which the same reasoning applies) appearing in the molecule's enviroment. Therefore, the updates in a molecule's environment are always shown in figures tagged by a boxed number identifying the affected node in the molecule's skeleton.

The choice of which dag is rerouted and which one gets arc bindings is arbitrary.

For reasons discussed later, the cost of looking up instance node updates in Boyer and Moore's environment representation is $O(|d|)$, where $|d|$ is the length of the derivation (a sequence of resolutions) of the instance. In the present representation, however, this cost is only $O(\log |d|)$. This better performance is achieved by particularizing the environment representation and by splitting the representational scheme into two components: a memory organization and a dag representation.

A dag representation is a way of mapping the mathematical entity dag onto a memory. A memory organization is a way of putting together a memory that has certain properties with respect to lookup, updating and copying. One can think of the memory organization as the hardware and the dag representation as the data structure.

\section{Memory organization}

In practice, random-access memory can be accessed and updated in constant time. However, updates destroy old values, which is obviously unacceptable when dealing with alternative updates of the same data structure. If we want to keep the old version, we need to copy it first into a separate part of memory and change the copy instead. For the normal kind of memory, copying time is proportional to the size of the object copied.

The present scheme uses another type of memory orgaaization - virtual-copy arrays - which requires $O(\log n)$ time to access or update an array with highest used index

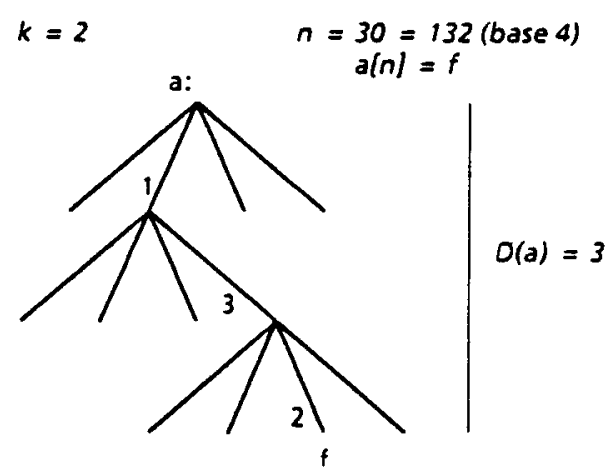

Figure 4: Virtual-Copy Array

of $n$, but in which the old contents are not destroyed by updating. Virtual-copy arrays were developed by David H. D. Warren [10] as an implementation of extensible arrays for Prolog.

Virtual-copy arrays provide a fully general memory structure: anything that can be stored in random-arress memory can be stored in virtual-copy arrays. although pointers in machine memory correspond to indexes in a virtual-copy array. An updating operation takes a virtual-copy array, an index, and a new value and returns a new virtual-copy array with the new value stored at the given index. An access operation takes an array and an index, and returns the value at that index.

Basically, virtual-copy arrays are $2^{k}$-ary trees for some fixed $k>0$. Define the depth $d(n)$ of a tree note $n$ to be 0 for the root and $d(p)+1$ if $p$ is the parent of $n$. Each virtual-copy array $a$ has also a positive depth $D(a) \geq \max \{d(n): n$ is a node of $a\}$. A tree node at depth $D(a)$ (necessarily a leaf) can be either an array element or the special marker $\perp$ for unassigned elements. All leaf nodes at depths lower than $D(a)$ are also $\perp$, indicating that no elements bave yet been stored in the subarray below the node. With this arrangement, the array can store at most $2^{k D(a)}$ elements, numbered 0 through $2^{k D(a)}-1$, but unused subarrays need not be allocated.

By numbering the $2^{k}$ daughters of a nonleaf node from $n$ to $2^{k}-1$, a path from $a^{\prime} s$ root to an array element (a leaf at depth $D(a))$ can be represented by a sequence $n_{0} \cdots n_{D(a)-1}$ in which $n_{d}$ is the number of the branch taken at depth $d$. This sequence is just the base $2^{k}$ representation of the index $\boldsymbol{n}$ of the array element, with $n_{0}$ the most significant digit and $n_{D(a)}$ the least significant (Figure 1).

When a virtual-copy array $a$ is updated, one of two things may happen. If the index for the updated element exceeds the maximum for the current depth (as in the $a[8]:=g$ update in Figure 5), a new root node is created for the updated array and the old array becomes the leftmost daughter of the new root. Other nodes are also created, as appropriate, to reach the position of the new element. If, on the other hand, the index for the update is within the range for the current 


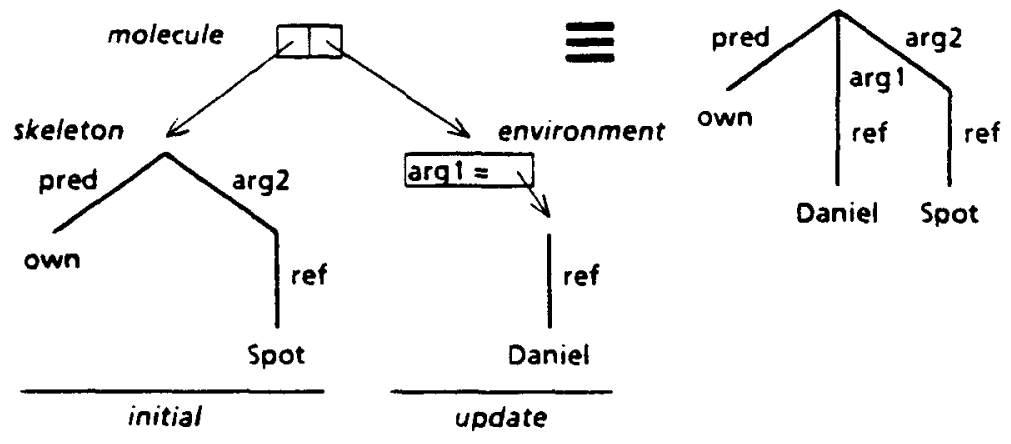

Figure 2: Molecule

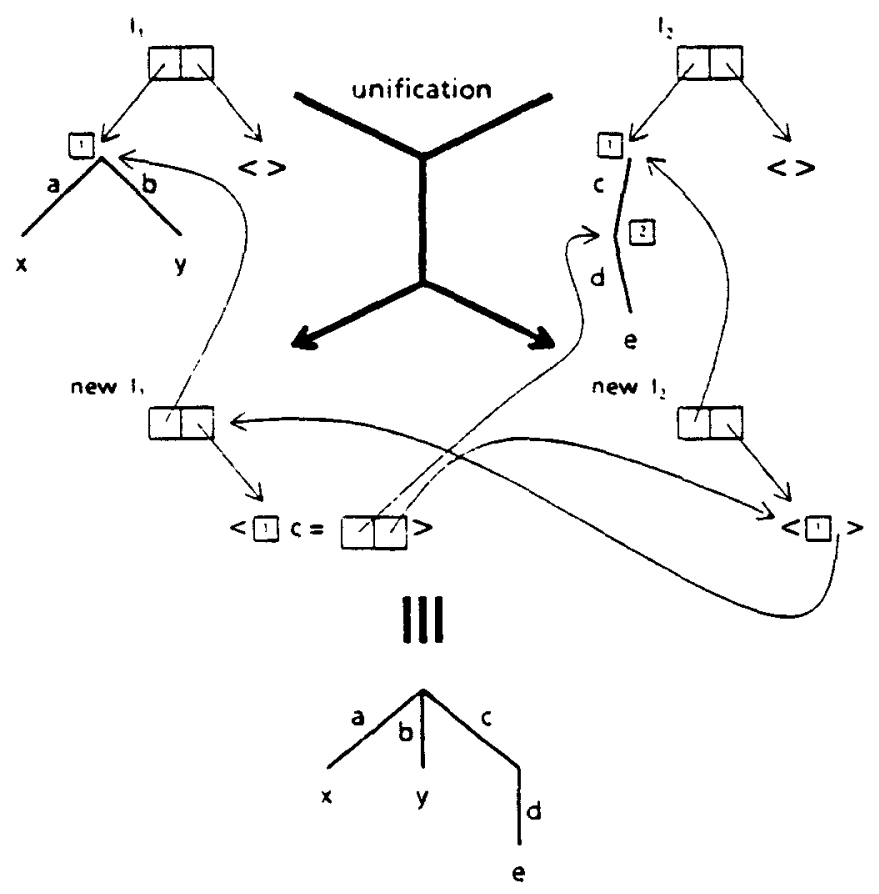

Figure 3: Unification of Two Molecules 


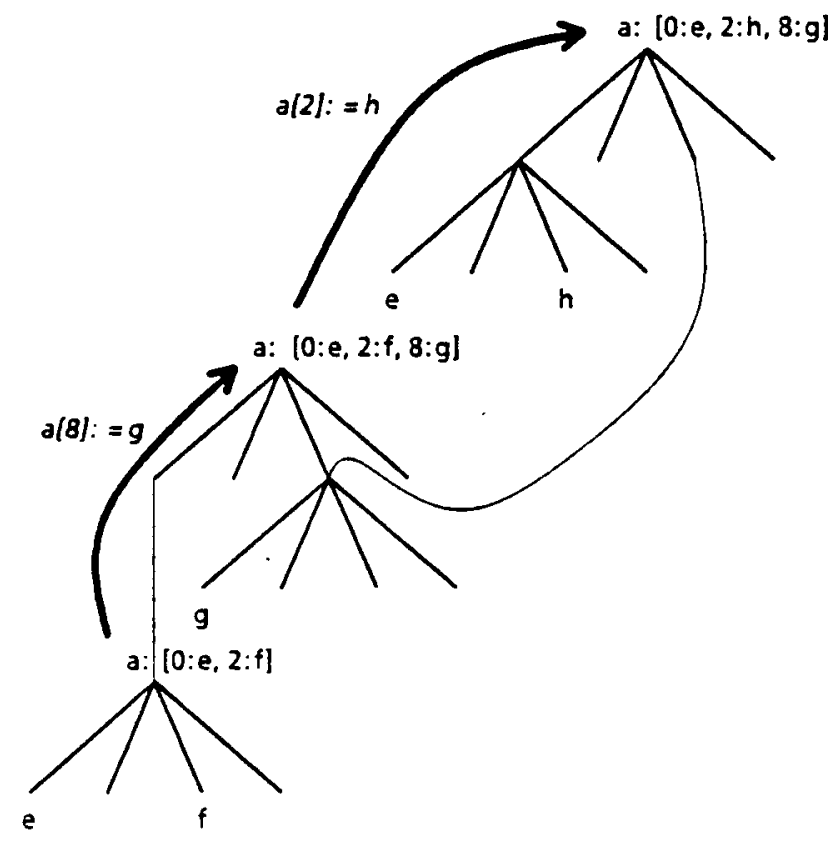

Figure 5: Updating Virtual-Copy Arrays

depth. the path from the root to the element being updated is copied and the old element is replaced in the new tree by the new element (as in the $a[2 \mid:=h$ update in Figure 5). This rescription assumes that the element being updated has already been set. If not, the branch to the element may imrninate prematurely in a $\perp$ leaf, in which case new nodes are rreated to the required depth and attached to the appropriate position at the end of the new path from the root.

\section{Dag representation}

Any dag representation can be implemented with virtualcopy memory instead of random-access memory. If that were done for the original PATR-II copying implementation, a certain measure of structure sharing would be achieved.

The present scheme, however, goes well beyond that by using the method of structure sharing introduced in Section 4. As we saw there, an instance object is represented by a molecule, a pair consisting of a skeleton dag (from a rule or iexical entry) and an update environment. We shall now examine the structure of environments.

In a chart parser for PATR-II, dag instances in the chart fall into two classes.

Base instances are those associated with edges that are created directly from lexical entries or rules.

Derived instances occur in edges that result from the combination oi a left and a right parent edge containing the left and right parent instances of the derived instance. The left ancestors of an instance (edge) are its left parent and that parent's ancestors, and similarly for right ancestors. I will assume, for ease of exposition, that a derived instance is always a subdag of the unification of its right parent with a subdag of its left parent. This is the case for most common parsing algorithms, although more general schemes are possible [7].

If the original Boyer-Moore scheme were used directly, the environment for a derived instance would consist of pointers to left and right parent instances, as well as a list of the updates needed to build the current instance from its parents. As noted before, this method requires a worst-case $O(|d|)$ search to find the updates that result in the current instance.

The present scheme relies on the fact that in the great majority of cases no instance is both the left and the right ancestor of another instance. I shall assume for the moment that this is always the case. In Section 9 this restriction will be removed.

It is a simple observation about unification that an update of a node of an instance $I$ is either an update of $I$ 's skeleton or of the value (a subdag of another instance) of another update of $I$. If we iterate this reasoning, it becomes clear that every update is ultimately an update of the skeleton of a base instance ancestor of $I$. Since we assumed above that no instance could occur more than once in I's derivation, we can therefore conclude that I's environment consists only of updates of nodes in the skeletons of its base instance ancestors. By numbering the base instances of a derivation consecutively, we can then represent an environment by an array of frames, each containing all the updates of the sketeton of a given base instance.

Actually, the environment of an instance $I$ will be a branch environment containing not only those updates directly relevant to $I$, but also all those that are relevant to the instances of I's particular branch through the parsing search space.

In the context of a given branch environment, it is then possible to represent a molecule by a pair consisting of a skeleton and the index of a frame in the environment. In particular, this representation can be used for all the values (dags) in updates.

More specifically, the frame of a base instance is an array of update records indexed by small integers representing the nodes of the instance's skeleton. An update record is either a list of arc bindings for distinct arc labels or a rerouting update. An arc binding is a pair consisting of a label and a molecule (the value of the arc binding). This represents an addition of an arc with that label and that value at the. given node. A reronting update is just a pointer to another molecule: it says that the subdag at that node in the updaterl dag is given by that molecule (rather than by whatever was in the initial skeleton).

To see bow skeletons and bindings work together to represent a dag, consider the operation of finding the subdag $d /\left\langle l_{1} \cdots l_{m}\right\rangle$ of $\operatorname{dag} d$. For this purpose, we use a current skeleton $s$ and a current frame $f$, given initially by the skeleton and frame of the molecule representing $d$. Now assume 
that the current skeleton $s$ and current frame $f$ correspond to the subdag $d^{\prime \prime}=d /\left\langle l_{1} \cdots l_{i-1}\right\rangle$. To find $d /\left\langle l_{1} \cdots l_{i}\right\rangle=d^{\prime} / l_{i}$, we use the following method:

1. If the top node of $s$ has been rerouted in $f$ to a dag $v$, dereference $d^{\prime}$ by setting $s$ and $f$ from $v$ and repeating this step; otherwise

2. If the top node of $g$ has an arc labeled by $l_{i}$ with value $s^{\prime}$, the subdag at $l_{i}$ is given by the moledule $\left(s^{\prime}, f\right)$; otherwise

3. If $f$ contains an are binding labeled $l_{i}$ for the top node of $s$, the subdag at $l_{i}$ is the value of the binding

If none of these steps can be applied, $\left\langle l_{1} \ldots l_{i}\right\rangle$ is not a path from the root in $d$.

The details of the representation are illustrated by the example in Figure 6, which shows the passive edges for the chart analysis of the string $a b$ according to the sample grammar

$$
\begin{aligned}
& S \rightarrow A B:\langle S a\rangle=\langle A\rangle \\
& \langle S b\rangle=\langle B\rangle \\
& \langle S a x\rangle=\langle S b y\rangle \\
& A \rightarrow a: \quad\langle A \cup v\rangle=a \\
& B \rightarrow b: \quad\langle B \cup v\rangle=b
\end{aligned}
$$

For the sake of simplicity, only the subdags corresponding to the explicit equations in these rules are shown (ie., the cat dag ares and the rule ares $0,1, \ldots$ are omitted). In the figure, the three nonterminal edges (for phrase types $S$, $A$ and $B$ ) are labeled by molecules representing the corresponding dags. The skeleton of each of the three molecules comes from the rule used to build the nonterminal. Each molecule points (via a frame index not shown in the figure) to a frame in the branch environment. The frames for the $A$ and $B$ edges contain arc bindings for the top nodes of the respective skeletons whereas the frame for the $S$ edge reroute nodes 1 and 2 of the $S$ rule skeleton to the $A$ and $B$ molecules respectively.

\section{The Unification Algorithm}

I shall now give the unification algorithm for two molecules (dags) in the same branch environment.

We can treat a complex dag $d$ as a partial function from labels to dags that maps the label on each are leaving the top node of the dag to the dag at the end of that arc. This allows us to define the following two operations between dags:

$$
\begin{aligned}
d_{1} \backslash d_{2} & =\left\{(l, d) \in d_{1} \mid l \notin \operatorname{dom}\left(d_{2}\right)\right\} \\
d_{1} \triangleleft d_{2} & =\left\{(l, d) \in d_{1} \mid l \in \operatorname{dom}\left(d_{2}\right)\right\}
\end{aligned}
$$

It is clear that $\operatorname{dom}\left(d_{1} \triangleleft d_{2}\right)=\operatorname{dom}\left(d_{2} \triangleleft d_{1}\right)$.

We also need the notion of dag dereferencing introduced in the last section. As a side effect of successive unifications, the top node of a dag may be rerouted to another dag whose top node will also end up being rerouted. Dereferencing is the process of following such chains of rerouting pointers to reach a dag that has not been rerouted.

The unification of dags $d_{1}$ and $d_{2}$ in environment $e$ consists of the following steps:

1. Dereference $d_{1}$ and $d_{2}$

2. If $d_{1}$ and $d_{2}$ are identical, the unification is immediately successful

3. If $d_{1}$ is a leaf, add to $e$ a rerouting from the top node of $d_{1}$ to $d_{2}$; otherwise

4. If $d_{2}$ is a leaf, add to $e$ a rerouting from the top node of $d_{2}$ to $d_{1}$; otherwise

5. If $d_{1}$ and $d_{2}$ are complex dags, for each arc $(l, d) \in d_{1} \triangleleft$ $d_{2}$ unify the dag $d$ with the dag $d^{\prime}$ of the corresponding arc $\left(l, d^{\prime}\right) \in d_{2} \triangleleft d_{1}$. Each of those unifications may add new bindings to $e$. If this unification of subdags is successful, all the arcs in $d_{1} \backslash d_{2}$ are are entured in $e$ as arc bindings for the top node of $d_{2}$ and finally the inp node of $d_{1}$ is rerouted to $d_{2}$.

6. If none of the conditions above applies, the unification fails.

To determine whether a dag node is a leaf or complex, both the skeleton and the frame of the corresponding molecule must be examined. For a dereferenced molecule. the set of arcs leaving a node is just the union of the skeleton arcs and the arc bindings for the node. For this to make sense, the skeleton arcs and arc bindings for any molecule node must be disjoint. The interested reader will have no difficulty in proving that this property is preserved by the unification algorithm and therefore all molecules built irom skeletons and empty frames by unification will satisfy it.

\section{Mapping dags onto virtual-copy memory}

As we saw above, any dag or set of dags constructed by the parser is built from just two kinds of material: (1) frames; (2) pieces of the initial skeletons from rules and lexical entries. The initial skeletons can be represented trivially by host language data structures, as they never change. Frames, though, are always being updated. A new frame is born with the creation of an instance of a rule or lexical entry when the rule or entry is used in some parsing step (uses of the same rule or entry in other steps beget their own frames). A frame is updated when the instance it belongs to participates in a unification.

During parsing, there are in general several possible ways of continuing a derivation. These correspond to alternative ways of updating a branch environment. In abstract terms, 


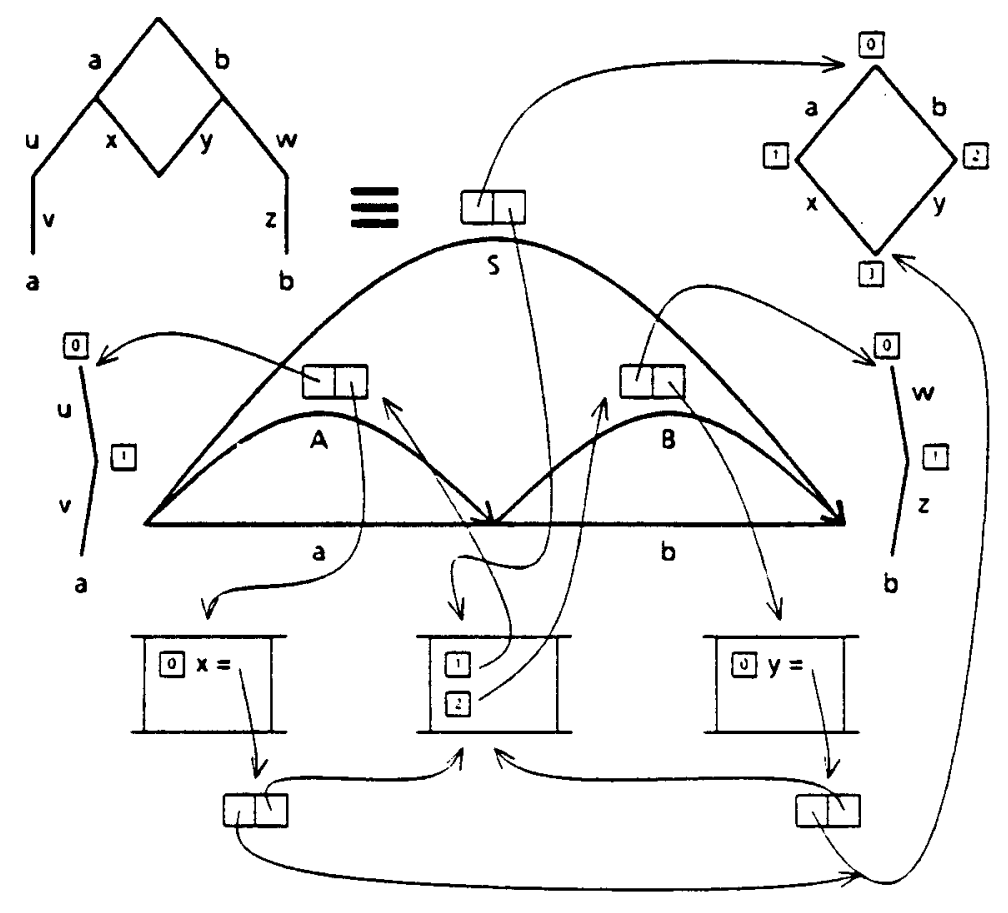

Figure 6: Structure-Sharing Chart

on coming to a choice point in the derivation with $\boldsymbol{n}$ possible continuations, $n-1$ copies of the environment are made, giving $n$ environments - namely, one for each alternative. In fact, the use of virtual-copy arrays for environments and frames renders this copying unnecessary, so each continuation path performs its own updating of its version of the environment without interfering with the other paths. Thus, all unchanged portions of the environment are shared.

In fact, derivations as such are not explicit in a chart parser. Instead, the instance in each edge has its own branch "nvironment, as described previously. Therefore. when two ages are combined, it is necessary to merge their environments. The cost of this merge operation is at most the same as the worst case cost for unification proper $(O(|d| \log |d|))$ However, in the very common case in which the ranges of frame indices of the two environments do not overlap, the merge cost is only $O(\log |d|)$.

To summarize, we have sharing at two levels: the BoverWoore style dag representation allows derived dag instances to share input data structures (skeletons), and the virtual-copy array environment representation allows different branches of the search space to share update records.

\section{The Renaming Problem}

In the foregoing discussion of the structure-sharing method, I assumed that the left and right ancestors of a derived instance were disjoint. In fact, it is easy to show that the con- dition holds wheneser the grammar does not allow empty derived eriges.

In contrast, it is possible to construrt a grammar in which an empty derived edge with dag $D$ is buth a left and a right ancestor of another erlge with dag $E$. ('learly, the two uses of $D$ as an ancestor of $E$ are mutually independent and the corresponding updates have to be segreogated. In other words, we need two copies of the instance D. By analegy: with theorem proving. I all this the remaming problem.

The current solution is to use real copying to turn the empty edge into a skeleton. which is then adeled to the chart The new skeleton is then used in the normal fashion to produce multiple instances that are free of mutual interference.

\section{Implementation}

The representation described here has been used in a PITRII parser implemented in Prolog. Two versions of the parser exist - one using an Farley-style algorithm related to Earley derfuction [7], the other using a left-corner algorithm.

Preliminary tests of the left-corner algorithm with struc. ture sharing on various grammars and input have shown parsing times as much as $60 \%$ faster (never less, in fact, than $40^{\circ} \%$ faster) than those achieved by the same parsing algorithm with structure copying. 


\section{References}

[1] R. S. Boyer and J S. Moore. The sharing of structure in theorem-proving programs. In Machine Intelligence 7, pages 101-116, John Wiley and Sons, New York, New York, 1972.

[2] J. Bresnan and R. Kaplan. Lexical-functional grammar: a formal system for grammatical representation. In J. Bresnan, editor, The Mental Representation of Grammatical Relations, pages 173-281, MIT Press, Cambridge, Massachusetts, 1982.

[3] M. Kay. Algorithm Sehemata and Data Structures in Syntactic Processing. Technical Report, XEROX Palo Alto Research Center, Palo Alto, California, 1980. A version will appear in the proceedings of the Nobel Symposium on Text Processing, Gothenburg, 1980.

[4] M. Kay. Functional grammar. In Proc. of the Fifth Annual Meeting of the Berkeley Linguistic Society, pages 142-158, Berkeley Linguistic Society, Berkeley, California, February 17-19 1979.

[5] Fernando C. N. Pereira and Stuart M. Shieber. The semantics of grammar formalisms seen as computer languages. In Proc. of Coling84, pages 123-129, Assoriation for Computational Linguistics, 1984.

(6) Fernando C. N. Pereira and David H. D. Warren. Definite clause grammars for language analysis - a survey of the formalism and a comparison with augmented transition networks. Artificial Intelligence, 13:231-278, 1080.

[7] Fernando C. N. Pereira and David H. D. Warren. Parsing as deduction. In Proe. of the 21st Annual Meeting of the Association for Ciomputational Linguistics. MIT, Cambridge, Massachusetts, June 15-17 1983.

[8] Stuart M. Shieber. The design of a computer language for linguistic information. In Proc. of Coling84, pages 362-366, Association for Computational tinguistics, 1984.

[9] David H. D. Warren. Applied Logic - its use and implementation as programming tool. $\mathrm{PhD}$ thesis, Iniversity of Edinburgh, Scocland, 1977. Reprinted as Twehniral Note 290, Artificial Intelligence Center, SRI, International, Menlo Park, California.

[10] David H. D. Warren. Logarithmic access arrays for Prolog. Unpublished program, 1983. 